\title{
Nuclear equation of state in the relativistic mean field model with density dependent coupling constants
}

\author{
Maria Voskresenskaya* \\ GSI Helmholtzzentrum für Schwerionenforschung GmbH, \\ Planckstraße 1, 64291 Darmstadt, Germany
}

E-mail: m.voskresenskaya@gsi.de

\section{Stefan Typel}

Excellence Cluster Universe, Technische Universität

München, Boltzmannstraße 2, 85748 Garching, Germany

GSI Helmholtzzentrum für Schwerionenforschung GmbH,

Planckstraße 1, 64291 Darmstadt, Germany

E-mail: s.typel@gsi.de

The description of neutron star matter requires the knowledge of the equation of state (EoS) in a wide range of densities. In this work we are trying to improve the phenomenological description of nuclear matter within a relativistic mean field (RMF) model with density dependent coupling constants. The couplings are well constrained only near the saturation density of nuclear matter and are extrapolated to smaller and higher densities. A comparison of the RMF equation of state of nuclear matter with the virial expansion leads to new constraints for the couplings at small densities. A new parametrization of density dependent couplings is suggested based on these constraints.

11th Symposium on Nuclei in the Cosmos

19-23 July 2010

Heidelberg, Germany.

\footnotetext{
*Speaker.
} 


\section{Introduction}

In neutron star physics one has to determine a reliable equation of state (EoS) of nuclear matter in a wide range of densities [1]. In astrophysical simulations of supernovae a wide range in temperatures and neutron-proton asymmetries has to be covered as well. Different models are used to provide a reasonable behavior for the EoS, valid in various density and temperature regions, however these models can hardly be united. Relativistic Mean Field (RMF) models, based on the Walecka model [2], describe nuclear matter below, above and around nuclear saturation density $\rho_{\text {sat }} \simeq 0.16 \mathrm{fm}^{-3}[3,4,5,6]$ using a quasiparticle approach.

But such models do not describe nuclear matter properties at densities $\rho \ll \rho_{\text {sat }}$ as well as models based on realistic nucleon-nucleon potentials constrained by scattering data in vacuum. At low densities and not too low temperatures one can consider a virial EoS [7], which gives a model-independent description of nuclear matter that takes two-body correlations into account using experimental information of nucleon-nucleon scattering phase shifts.

Here we consider a RMF model with density dependent couplings [5] where the coupling functions were determined from fits to properties of finite nuclei. The model was recently extended to include the formation of clusters at low densities [8]. The aim of this work is to modify the density dependence of the couplings at very low densities in order to match the virial EoS and, if possible, to give a reasonable description of nucleon-nucleon scattering phase shifts.

This paper is organized as follows. In Section 2 we present the parametrisation of the mesonnucleon couplings in the RMF model [5]. Constraints from the virial expansion are discussed in Section 3. Concluding remarks and an outlook are given in Section 4.

\section{RMF model with couplings depending on the density}

We start with a RMF model with density dependent coupling constants $\Gamma_{l}$ of four mesons $l=\sigma, \omega, \rho, \delta$ with masses $m_{l}$ that are commonly considered in this approach to model the nucleonnucleon $(\mathrm{NN})$ interaction [5]. The coupling functions are taken in the form:

$$
\Gamma_{l}(\rho)=\Gamma_{l}\left(\rho_{\mathrm{sat}}\right) f_{l}(x), \quad x=\frac{\rho}{\rho_{\mathrm{sat}}}
$$

with the nuclear matter density $\rho$ and the functions $f_{l}(x)$ considered in [5]. In [5] $\Gamma_{\delta}$ was put to zero. As we argue in Sect. 4, we need $\Gamma_{\delta} \neq 0$ to match the RMF EoS with the virial limit at low densities. Previously, parameters of the model were fitted to properties of finite nuclei, now we would like to modify the coupling functions at densities $\rho \ll \rho_{\text {sat }}$ to fulfill the virial limit and NN scattering data.

\section{Constraining the coupling constants from the virial expansion}

The virial equation of state presents a model independent approach in the calculation of thermodynamical properties of low density matter, provided the fugacities $z_{i}=\exp \left(\frac{\mu_{i}}{T}\right)$ of the particles $i$ with nonrelativistic chemical potential $\mu_{i}$ are small. The range of densities where the virial expansion is valid can be estimated by the relation $\rho_{i} \lambda_{i}^{3} \ll 1$ where $\lambda_{i}=\left(2 \pi / m_{i} T\right)^{1 / 2}$ is the thermal 
wave length at temperature $T$ and $m_{i}$ is the particle mass. The pressure $P$ of the matter is given by the logarithm of the grand canonical partition function, which is expanded in a power series of fugacities:

$$
P=T \frac{\log \Omega}{V}=T\left(\sum_{i} g_{i} \frac{z_{i}}{\lambda_{i}^{3}}+\sum_{i j} b_{i j} \frac{z_{i} z_{j}}{\lambda_{i}^{\frac{3}{2}} \lambda_{j}^{\frac{3}{2}}}\right) .
$$

Particle degeneracy factors are denoted by $g_{i}$ and $b_{i j}$ are the second virial coefficients given by

$$
b_{i j}=\frac{1+\delta_{i j}}{2} \frac{\lambda_{i}^{\frac{3}{2}} \lambda_{j}^{\frac{3}{2}}}{\lambda_{i j}^{3}} \int d E \exp \left(-\frac{E}{T}\right)\left[\sum_{k} g_{k}^{i j} \delta\left(E-E_{k}^{i j}\right)+\sum_{l} \frac{g_{l}^{i j}}{\pi} \frac{d \delta_{l}^{i j}}{d E}\right] \pm \delta_{i j} g_{i} 2^{-\frac{5}{2}}
$$

with $\lambda_{i j}=\left(2 \pi /\left(m_{i}+m_{j}\right) T\right)^{1 / 2}$. The quantities $g_{k}^{i j}$ and $g_{l}^{i j}$ denote the degeneracy factor for bound states $k$ with energy $E_{k}^{i j}<0$ and scattering states in channel $l$ with phase shifts $\delta_{l}^{i j}(E)$, respectively. The term $\pm \delta_{i j} g_{i} 2^{-\frac{5}{2}}$ accounts for the correction for Bose-Einstein or Fermi-Dirac statistics. Neutron and proton densities then have the form:

$$
n_{n}=\left(g_{n} \frac{z_{n}}{\lambda_{n}^{3}}+2 b_{n n} \frac{z_{n}^{2}}{\lambda_{n}^{3}}+2 b_{n p} \frac{2 z_{n} z_{p}}{\lambda_{n}^{\frac{3}{2}} \lambda_{p}^{\frac{3}{2}}}\right), n_{p}=\left(g_{p} \frac{z_{p}}{\lambda_{p}^{3}}+2 b_{p p} \frac{z_{p}^{2}}{\lambda_{p}^{3}}+2 b_{p n} \frac{2 z_{p} z_{n}}{\lambda_{p}^{\frac{3}{2}} \lambda_{n}^{\frac{3}{2}}}\right)
$$

The energy dependence of the phase shift can be expressed in terms of scattering length $a_{l}^{i j}$ using the effective range expansion. For $S$-waves one has $k \cot \left(\delta_{l}^{i j}\right)=-\frac{1}{a_{l}^{i j}}, k=\sqrt{2 m_{i j} E}$ and $m_{i j}=\frac{m_{i} m_{j}}{m_{i}+m_{j}}$. The virial coefficients can be calculated analytically. With $a_{t}^{n p}>0, a_{s}^{n p}<0$ and $a_{s}^{n n}<0$ one obtains in the limit $T \rightarrow 0$ the approximations

$$
\begin{aligned}
& b_{n n} \approx \frac{\lambda_{n}^{3}}{2 \sqrt{\pi} \lambda_{n n}^{3}} \sqrt{2 m_{n n} T\left[a_{s}^{n n}\right]^{2}}-2^{-\frac{3}{2}} \\
& b_{n p} \approx \frac{\lambda_{n}^{\frac{3}{2}} \lambda_{p}^{\frac{3}{2}}}{2 \lambda_{n p}^{3}}\left[\frac{1}{2 \sqrt{\pi}} \sqrt{2 m_{n p} T\left[a_{s}^{n p}\right]^{2}}-\frac{3}{2 \sqrt{\pi}} \sqrt{2 m_{n p} T\left[a_{t}^{n p}\right]^{2}}+3 \exp \left(\frac{B_{d}}{T}\right)\right],
\end{aligned}
$$

where $B_{d}$ is the deuteron binding energy. Now let us consider generalized RMF description [8] with neutrons, protons and deuterons as degrees of freedom. The density of neutrons and protons is:

$$
n_{i}=g_{i} \int \frac{d^{3} k}{(2 \pi)^{3}}\left\{\exp \left[\frac{1}{T}\left(V_{i}+\sqrt{k^{2}+\left(m_{i}^{*}\right)^{2}}-\tilde{\mu}_{i}\right)\right]+1\right\}^{-1},
$$

where $\tilde{\mu}_{i}$ is the relativistic chemical potential, $m_{i}^{*}=m_{i}-S_{i}$ is the effective mass and $V_{i}, S_{i}$ are vector and scalar potentials. The density of the deuterons is given by a similar expression with the Bose-Einstein distribution function. We perform an expansion in powers of the neutron and proton fugacities keeping only contributions linear or quadratic in $z_{i}$. Finally, a comparison of the RMF results with the virial expression of the densities for $T \rightarrow 0$ leads to the relations:

$$
\begin{aligned}
& \frac{\Gamma_{\delta}^{2}}{m_{\delta}^{2}}-\frac{\Gamma_{\rho}^{2}}{m_{\rho}^{2}}=-\pi\left[\frac{a_{s}^{n n}}{m_{n}}-\frac{m_{n}+m_{p}}{m_{n} m_{p}} \frac{a_{s}^{n p}+3 a_{t}^{n p}}{4}\right] \\
& \frac{\Gamma_{\sigma}^{2}}{m_{\sigma}^{2}}-\frac{\Gamma_{\omega}^{2}}{m_{\omega}^{2}}=-\pi\left[\frac{a_{s}^{n n}}{m_{n}}+\frac{m_{n}+m_{p}}{m_{n} m_{p}} \frac{a_{s}^{n p}+3 a_{t}^{n p}}{4}\right]
\end{aligned}
$$




\begin{tabular}{|l|l|l|l|l|l|l|l|}
\hline$\Gamma_{\sigma}(0)$ & $\Gamma_{\omega}(0)$ & $\Gamma_{\rho}(0)$ & $\Gamma_{\delta}(0)$ & $m_{\sigma}$ & $m_{\omega}$ & $m_{\rho}$ & $m_{\delta}$ \\
\hline 11.32017 & 15.96944 & 10.45724 & 13.47244 & $550 \mathrm{MeV}$ & $783 \mathrm{MeV}$ & $763 \mathrm{MeV}$ & $983 \mathrm{MeV}$ \\
\hline
\end{tabular}

Table 1: Coupling constants at zero density and masses of the mesons.

where the coupling constants have to be taken at zero density. In model [5] the coupling constants at very small densities are not well constrained and simply follow from the extrapolation with the assumed functions $\Gamma_{l}(\rho)$. Now we use relations (3.7) and (3.8) to constrain the couplings at low densities. Simultaneously, we try to fit $\Gamma_{l}(0)$ to describe NN scattering phase shift at low densities including the pion in the boson exchange potential that gives no contribution in the mean-field approximation for nuclear matter. However, it was rather difficult to find a good parametrization for the couplings in the low-density EoS and the NN scattering simultaneously. Therefore we suggest to incorporate not only the deuteron bound state but also the two-body scattering correlations in the generalized RMF model. Continuum contributions can be represented effectively by resonances and treated like additional clusters with temperature depending energies. In this case the relations for the zero-density couplings assume the form:

$$
\frac{\Gamma_{\delta}^{2}}{m_{\delta}^{2}}-\frac{\Gamma_{\rho}^{2}}{m_{\rho}^{2}}=0 \quad \frac{\Gamma_{\sigma}^{2}}{m_{\sigma}^{2}}-\frac{\Gamma_{\omega}^{2}}{m_{\omega}^{2}}=0
$$

Respecting these relations, we are able to obtain the zero density coupling constants with a descripton of low-energy NN scattering. Results for the couplings $\Gamma_{l}(0)$ are given in Table 1 . In order to achieve a smooth transition from the previous density dependent couplings at high densities to the newly derived values of the couplings at zero density we suggest to use a modified version of the coupling functions by introducing

$$
\Gamma_{l}^{\text {new }}(\rho)=\left[1+\frac{\Gamma_{l}(0)^{\text {new }}-\Gamma_{l}(0)^{\text {old }}}{\Gamma_{l}(0)^{\text {old }}} \exp \left(-\alpha x^{2}\right)\right] \Gamma_{l}^{\text {old }}(\rho)
$$

This modification contains a parameter $\alpha \gg 1$ which governs the location of the transition point. $\Gamma_{l}(0)^{\text {new }}$ are the new coupling constants from Table 1 and $\Gamma_{l}(0)^{\text {old }}$ are the constants from the old parametrization. For the $\delta$ meson we assume the simple function $\Gamma_{l}^{\text {new }}(\rho)=\Gamma_{l}^{\text {new }}(0) \exp \left(-\alpha x^{2}\right)$. The form of the functions and the parameter $\alpha$ are chosen in such a way to reproduce the virial limit at low densities and to match with the old functions (fitted to the properties of finite nuclei) at high densities, where the virial expansion is no longer valid. In Fig.3 the modification of the coupling functions at low densities, as required by the new constraints, is depicted for a value of $\alpha=1600$. We observe a smooth transition at very low densities around the transition density $\rho_{\text {sat }} / \sqrt{\alpha} \approx 0.004 \mathrm{fm}^{-3}$.

A more general microscopic description of cluster formation and dissolution in dense matter was presented in [9]. This approach leads to generalized Beth-Uhlenbeck formula with contributions from bound and scattering states, however calculated from the in-medium T-matrix. We restricted ourselves to a more phenomenological approach that reproduces the standard virial EoS at low densities. 


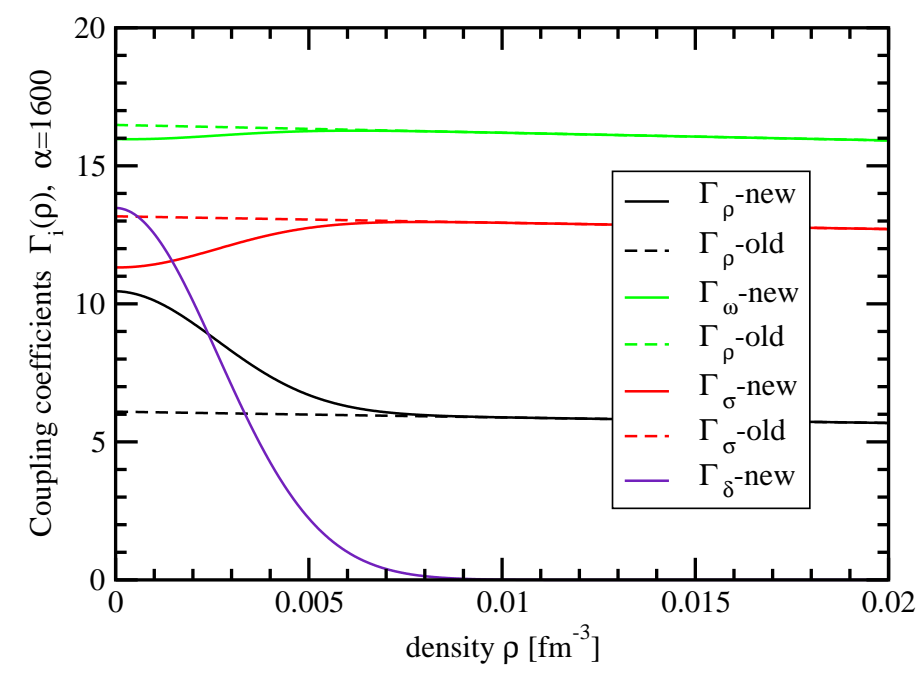

Figure 1: Density dependence of the meson-nucleon couplings in the original RMF model (dashed lines) and in the proposed form with a low-density modification (solid lines).

\section{Summary and outlook}

In this paper we have studied new constraints for the low density meson-nucleon couplings in a generalized RMF model that were derived by requiring a consistency of the RMF EoS with the virial equation of state at small densities, low temperatures and low-energy nucleon-nucleon scattering data. New parametrization functions for the coupling coefficients were proposed, taking into account these constraints. In the future we plan to implement these results in the calculation of the EoS in the generalized RMF model with bound state and scattering correlations and to study the effects on the thermodynamical properties of low-density matter. Finally the derived EoS will be applied in astrophysical model calculations, in particular in the study of neutron star properties and later in supernova simulations.

\section{References}

[1] T. Klahn et al, Phys. Rev. C74, 035802, 2006.

[2] J.D. Walecka, Ann. Phys. (N.Y.) 83 (1974) 491;

B.D. Serot, and J.D. Walecka, Adv. Nucl. Phys. 16, 1 (1996).

[3] B.D. Serot, and J.D. Walecka, Int. J. Mod. Phys. E 6, 515 (1997).

[4] Y.K. Gambhir, P. Ring, A. Thimet, Annals Phys. 198, 132 (1990).

[5] S. Typel, H.H. Wolter, Nucl.Phys. A656, 331 (1999); S. Typel, T. von Chossy, H. Wolter, Phys. Rev. C67, 034002 (2003); S.Typel, Phys. Rev. C71, 064301 (2005).

[6] E.E. Kolomeitsev and D.N. Voskresensky, Nucl. Phys. A759, 373 (2005).

[7] C.J. Horowitz, A. Schwenk, Phys. Lett. B638 153 (2006); Nucl. Phys. A776, 55 (2006). C.J. Horowitz, A. Schwenk, Phys. Lett. B642, 326 (2006).

[8] S. Typel et al, Phys. Rev. C81, 015803, 2010.

[9] M. Schmidt, G. Röpke and H. Schulz, Ann. Phys. 202, 57 (1990) 